\title{
Axillary sinuses and cervical swellings
}

\author{
S. Flann, S. Diaz-Cano, E. Higgins and D. Creamer
}

Department of Dermatology, King's College Hospital, London, UK

\section{Clinical findings}

A 22-year-old man was referred with a 3-year history of bilateral axillary lesions and an 18-month history of cervical swelling. The patient also complained of weight loss, anorexia, lethargy and intermittent fevers. Examination revealed two tender, fluctuant masses on the left side of the neck with overlying erythema. In addition, there were sinuses in both axillae (Fig. 1a,b). The patient was a refugee from Afghanistan but had been in the UK for the past 3 years. He had no other medical problems and was not on any regular medication.

Full blood count, renal and liver profiles, and erythrocyte sedimentation rate were normal and a human immunodeficiency virus (HIV) test was negative. Microbiological analysis of axillary pus swabs were negative, and chest X-ray was normal. Further investigations included a computed tomography scan of the chest, abdomen and pelvis, which revealed apical nonfibrotic lung nodules and extensive axillary lymphadenopathy (Fig. 1c). A biopsy of the cervical swelling was sent for histological and microbiological analysis. An incisional skin biopsy from the left axillary mass was also obtained for histological and microbiological examination. The initial differential diagnosis included lymphoma.

\section{Histopathological findings}

Histology of the axillary skin biopsy showed an intact epidermis and a necrotizing granuloma in the deep dermis with a moderate lymphocytic infiltrate (Fig. 2a,b). Special stains (Wade-Fite, Ziehl-Neelsen, Giemsa, DPAS) showed no acid-fast bacilli, parasites

Correspondence: Dr Sandy Flann, Department of Dermatology, King's College Hospital, London SE5 9RS, UK.

E-mail: sandyflann@hotmail.com

Conflict of interest: none declared.

Accepted for publication 25 October 2005 or fungi. Histology of the cervical swelling showed dense fibrous connective tissue with a chronic inflammatory cell infiltrate composed of mononuclear cells.

What is your diagnosis?
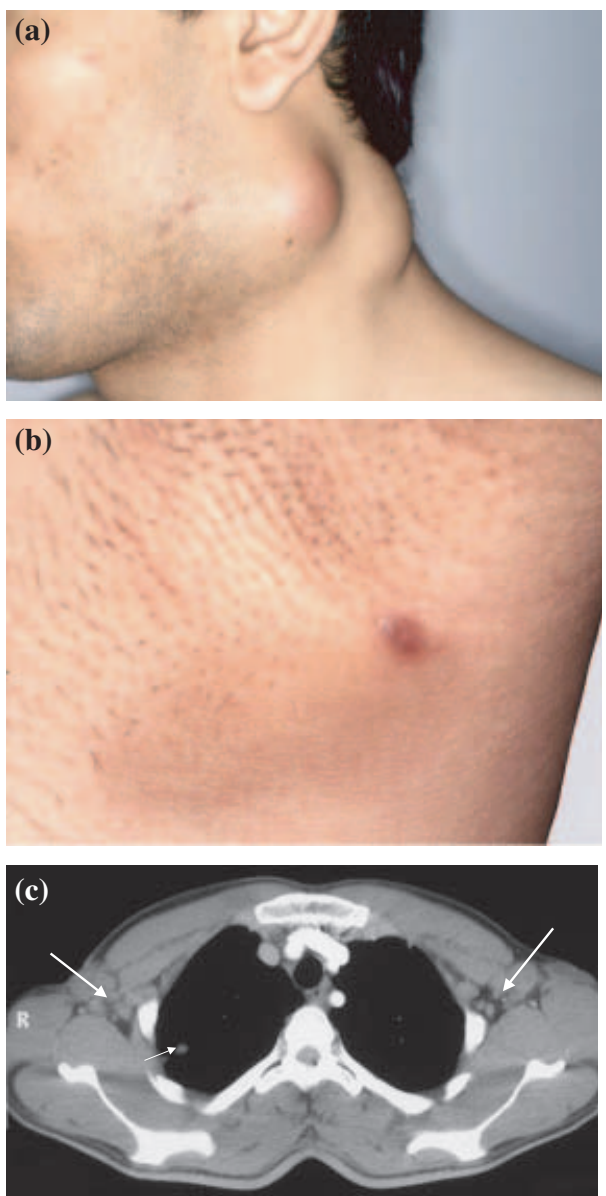

Figure 1 (a) Two tender, fluctuant masses on the left side of the neck with overlying surface erythema; (b) a discharging sinus in the left axilla; (c) chest CT scan demonstrates apical nonfibrotic nodules in the parenchyma of both lungs (small arrow). There is extensive axillary lymphadenopathy (large arrows). 

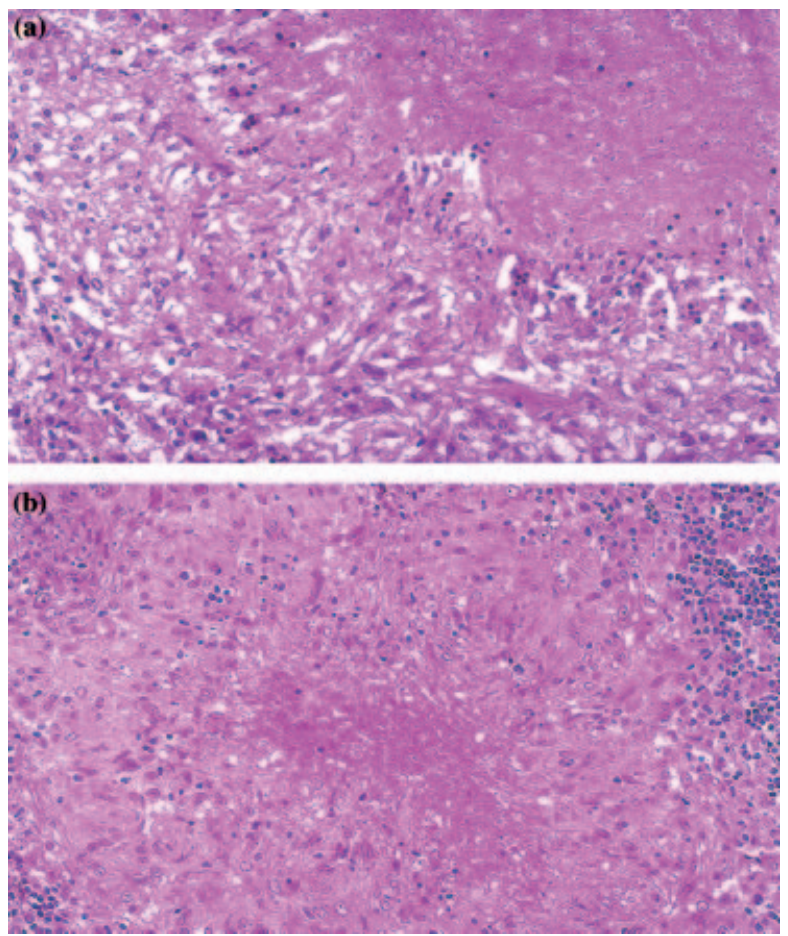

Figure 2 (a) Histopathology of skin and subcutaneous tissue. There is a solitary necrotizing granuloma in the deep dermis with a modest associated lymphocytic infiltrate. Ziehl-Neelsen staining was negative. (b) High-power view of granuloma with caseation necrosis. Haematoxylin and eosin; original magnification (a) $\times 100$; (b) $\times 200$.

\section{Diagnosis}

Scrofuloderma with active pulmonary tuberculosis.

\section{Discussion}

After 6 weeks, cultures of the cervical swelling biopsy and left axillary pus isolated Mycobacterium tuberculosis. The patient received antituberculous therapy (rifampicin, isoniazid, pyrazinamide, ethambutol) for 6 months, which resulted in full cutaneous and respiratory resolution.

The presentation of tuberculosis in the skin is varied, but scrofuloderma is rare, with an incidence varying from $0.03 \%$ to $0.15 \%$ in countries with a high tuberculosis prevalence. ${ }^{1,2}$ The term scrofula is derived from the Latin expression 'scroful' meaning 'swelling of the glands of the neck'. It occurs due to breakdown of skin overlying a tuberculous focus, usually a lymph node, but can also occur over infected bones in tuberculous osteomyelitis or joints in tuberculous arthritis. Prior to pasteurization, scrofuloderma was associ- ated with Mycobacterium bovis cervical adenitis. It presents typically as a discharging sinus over significant cervical lymphadenopathy.

The treatment of scrofuloderma is the same as that for pulmonary tuberculosis, and requires multidrug regimens because of increased drug resistance. ${ }^{3}$ For drugsusceptible strains in HIV-seronegative patients, the treatment should last 6 months. The evidence for the role of surgery in the treatment of scrofuloderma is limited. On occasion, excision of scrofuloderma with the underlying focus may be required.,

\section{Learning points}

- Scrofuloderma is a rare presentation of cutaneous tuberculosis in the UK.

- Scrofuloderma presents typically as a discharging sinus over significant cervical lymphadenopathy.

- Scrofuloderma is caused by contiguous spread of TB infection from underlying tissue through the overlying subcutis and skin. It is important to consider tuberculosis in the differential diagnosis of skin lesions, as tuberculosis prevalence is increasing due to its association with HIV infection and also increased immigration from countries where tuberculosis is common.

- The management of scrofuloderma is medical, with some evidence for the role of surgery in recalcitrant cases.

\section{References}

1 Kumar B, Muralidhar S. Cutaneous tuberculosis: a twentyyear prospective study. Int J Tuberc Lung Dis 1999; 3: 494500.

2 Sehgal VN, Srivastava G, Khurana VK et al. An appraisal of epidemiologic, clinical, bacteriologic, histopathologic, and immunologic parameters in cutaneous tuberculosis. Int $J$ Dermatol 1987; 26: 521-6.

3 Joint Tuberculosis Committee of the British Thoracic Society. Chemotherapy and management of tuberculosis in UK. Recommendations 1998 .Thorax 1998; 53: 536-48.

4 Moergel M, Walter C, Coerdt W et al. [Chronic cutaneous infiltration with abscess and fistula formation. A type of clinical course in atypical mycobacteriosis.] Mund Kiefer Gesichtshir 2004; 8: 311-15.

5 Kaur S, Thami GP, Gupta PN et al. Recalcitrant scrofuloderma due to rib tuberculosis. Pediatric Dermatol 2003; 20: 309-12. 